\title{
Cirugía de la catarata con implante de lente intraocular y corrección del astigmatismo post-operatorio
}

\author{
Cataract surgery with ocular lens implantation and correction of postoperative
}

\section{CORDERO Raúl ${ }^{1}$}

${ }^{1}$ Especialista en Oftalmología. Instituto Nacional de Enfermedades Neoplásicas.

\section{SUMMARY}

We performed 20 cataract extracapsular extractions with intraocular lens implantation. $62 \%$ of the patients achieved a visual ......of $20 / 40$ or better. The corrective measures to control post-operative astigmatism were able to reduce it, in $79 \%$ of the cases, to 2 diopters or less. Intraocular lens implantation after catarac extraction offer the patient a satisfactory visual outcome, much better than the one achieved with conventional glasses. The post-operative astigmatism correction greatly reduces the post-operative astigmatism. (Rev Med Hered 1994; 5: 86-90).

KEY WORDS: Cataract, Post-op Astimastism, Intraocular lens.

\section{RESUMEN}

Se realizaron 29 extracciones extracapsulares de catarata con implante primario de lente intraocular. El $62 \%$ de los pacientes obtuvieron una agudeza visual de 20/40 o mejor. Las medidas correctivas para reducir el astigmatismo post-operatorio efectivo en el $79 \%$ de los casos, de 2 dioptrías o menos. El implante de lente intraocular primario le brindan al paciente con catarata una recuperación visual satisfactoria, mejor que la que se obtiene con la corrección óptica convencional. La corrección astigmática postoperatoria reduce considerablemente el astigmatismo postoperatorio. (Rev Med Heredia 1994; 5: 86-90).

PALABRAS CLAVE: Catarata, astigmatismo Post-operatorio, lentes intraoculares.

\section{INTRODUCCIÓN}

En los últimos años, la técnica de elección en la gran mayoría de operaciones de catarata ha sido la extracción extracapsular. La ventaja de esta técnica sobre la extracción intracapsular es que se deja una barrera natural que separa el vítreo de la cámara anterior y del humor acuoso, permitiendo la introducción de un lente intraocular de cámara posterior que ocupa, anatómicamente, el lugar del cristalino, disminuyendo la posibilidad del edema macular cistoide y de endoftalmitis, así como la 
descompensación corneal por contacto vítreo endotelial y el glaucoma por bloqueo pupilar causado por el vítreo.

El presente trabajo prospectivo da a conocer los resultados de agudeza visual y astigmatismo post operatorio, en pacientes operados de catarata con implante primario de lente intraocular, utilizando la técnica extracapsular.

Las cirugías fueron realizadas por el autor en su Tercer año de Residencia en la especialidad de Oftalmología de la Universidad Peruana Cayetano Heredia (sede Hospital Arzobispo Loayza).

\section{MATERIAL Y MÉTODOS}

Se incluyeron en el estudio 29 pacientes con catarata senil que fueron sometidos a extracción extracapsular de la catarata con implante primario de lente intraocular.

El criterio de inclusión fue el de presentar una agudeza visual, con su mejor corrección, de 20/70 o menos y no presentar alteraciones evidentes en el examen pre-operatorio.

Evaluación pre-operatoria:

A todos los pacientes se les midió la agudeza visual pre-operatoria sin corrección, con corrección y con agujero estenopeico.

La función macular se evaluó mediante la percepción de colores (rojo y verde).

La retina periférica se estudió mediante la ubicación de un estímulo luminoso en los cuatro cuadrantes del campo visual del ojo comprometido.

A todos los pacientes se les dilató la pupila con Tropicamida al 1\% para evaluar la opacidad del cristalino con mayor facilidad, detectar la presencia de sinequias y evaluar lo mejor posible el estado de la retina.

La determinación del poder dióptrico del lente intraocular se realizó en el A Scan Ultra 20/20 Storz.

Técnica Quirúrgica

En el pre-operatorio los pacientes recibían acetazolamida $(250 \mathrm{mg}$ la noche antes de la operación y $250 \mathrm{mg}$ media hora antes de la operación) y diazepan (10 mg media hora antes de la operación), vía oral.

Treinta minutos antes de la operación se les instilaba tropicamida al $1 \%$, fenilefrina al $2.5 \%$ ciclopentolato al $1 \%$ para conseguir una midriasis máxima.

En todos los pacientes se usó anestesia local retrobular y bloqueo palpebral según la técnica de O'brien.

Se fijo el recto superior y se usó el blefaróstato para abrir la hendidura palpebral. Se realizó un flap conjuntival de 180 grados con base en el fórnix, luego se hizo el surco esclero-corneal de 160 grados, y se punzó la cámara anterior con una aguja $\mathrm{N}^{\circ} 21$. 
Se procedió a la capsulotomía anterior en 360 grados para posteriormente abrir la incisión en su totalidad. Acto seguido se extrajo el núcleo del cristalino mediante presión contrapresión, suturándose luego la incisión en forma parcial. Se hizo la aspiración de los restos corticales con la cánula de Simcoe.

Seguidamente se insertó el lente de cámara posterior en la bolsa capsular o en el surco ciliar y se procedió a completar la sutura de la herida operatoria con puntos separados de nylon 10/0, previa iridectomía periférica en los casos que lo requirieron.

En el punto post-operatorio se les indicó una suspensión oftálmica de antibióticos y corticoesteroides y un anti-inflamatorio sistémico no-esteroideo. Estos se iban descontinuando de acuerdo a la respuesta inflamatoria del ojo.

Los pacientes fueron dados de alta al día siguiente de la operación y se les controló al segundo día post-operatorio para evaluar la reacción inflamatoria y la presencia del reflejo rojo retinal, parámetros importantes para descartar alguna complicación infecciosa y el peligro de una endoftalmitis (15).

Control del astigmatismo post-operatorio:

La primera lectura queratométrica se realizó entre la cuarta y quinta semana postoperatoria, programándose de esta manera el posible corte de suturas para la sexta semana.

Si el paciente presentaba una astigmatismo de 3 a 4 dioptrías, en la sexta semana se cortaba la sutura que estaba mas cerca de la lectura queratométrica más curva (más positiva) $(1,3)$ y se le citaba a la siguiente semana repitiéndose el procedimiento hasta obtener un astigmatismo de 2 dioptrías o menos.

Si el astigamtismo era mayor de 4, también se cortaba la sutura en el eje mas curvo, pero se le re-examinaba al día siguiente, cortándose una sutura más si era necesario. Si no se constaba una reducción evidente del astigmatismo al cabo de una hora del corte, se le citaba la semana siguiente y se repetía el procedimiento.

El corte de la sutura se realizó con una aguja $\mathrm{N}^{\circ} 22$ en el borde corneal de la sutura (1). De esta manera, el nylon que quedó hacia la córnea se introduce en ella y el otro cabo se desliza por debajo de la conjuntiva. Por otro lado se evita un sangrado innecesario.

El corte de suturas se realizó entre la sexta y octava semana postoperatoria $(1,12)$.

La refracción y la queratometría final se realizaron en la octava o novena semana postoperatoria.

\section{RESULTADOS}

Entre junio de 1989 y mayo de 1990 el autor realizó 32 operaciones de catarata con implante de lente intraocular de las que sólo incluímos 29, pués 3 no regresaron para el seguimiento respectivo. 
Quince pacientes (52\%) fueron del sexo femenino y 14 (48\%) del sexo masculino. Las edades fluctuaron entre los 52 y 85 años, siendo el promedio 68.3 años. Trece casos $(45 \%)$ fueron del ojo derecho y $16(55 \%)$ del izquierdo.

Agudeza visual

La agudeza visual pre-opeatoria varió desde 20/70 hasta solo percepción de luz. A los 2 meses postoperatorio $86 \%$ tenían agudeza visual $20 / 70$ o más y $62 \%$, 20/40 o más. (Cuadro $\mathrm{N}^{\mathrm{0}} 1$ ). De los 11 casos cuya agudeza visual fue menos de 20/40, 6 presentaban patología retinal (55\%). Tres de ellos tenían degeneración macular relacionada a la edad, 2 tenían agujero macular y 1 presentaba glaucoma con cambios severos en el nervio óptico. Los 4 casos con agudeza visual menor de 20/70 presentaban patología retinal: 2 con agujero macular, 1 con degeneración macular senil y 1 con glaucoma senil y 1 con glaucoma.

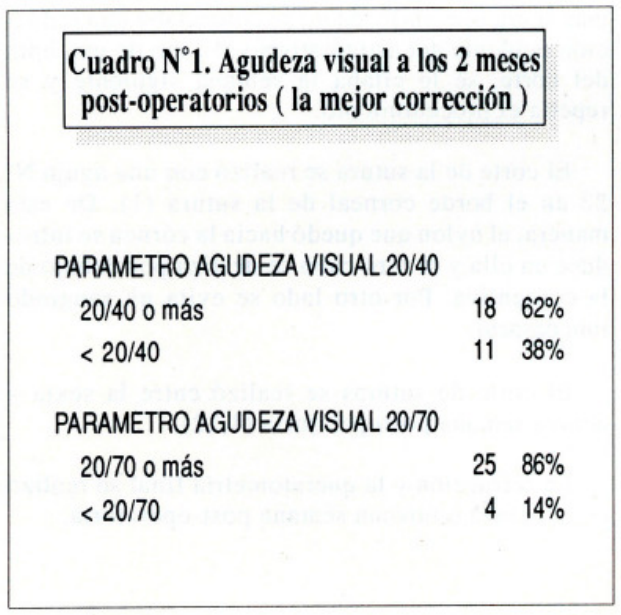

Astigmatismo post-operatorio

El astigmatismo post-operatorio más alto fue de 5.75 dioptrías. El 79\% de los pacientes tuvieron astigmatismo $<2$ dioptrias, a los 2 meses post-operatorio (Cuadro $\mathrm{N}^{\mathrm{o}} 2$ ).

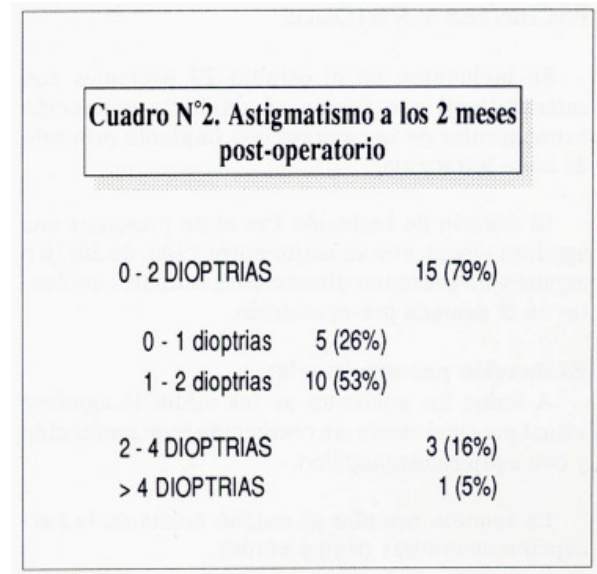

Complicaciones 
Las complicaciones intra-operatorias fueron 5, tal como se puede observar en el cuadro $\mathrm{N}^{\mathrm{o}}$ 3. Estas complicaciones intra-operatorias fueron manejadas en forma adecuada y se logró completar la operación con implante de lente intraocular.

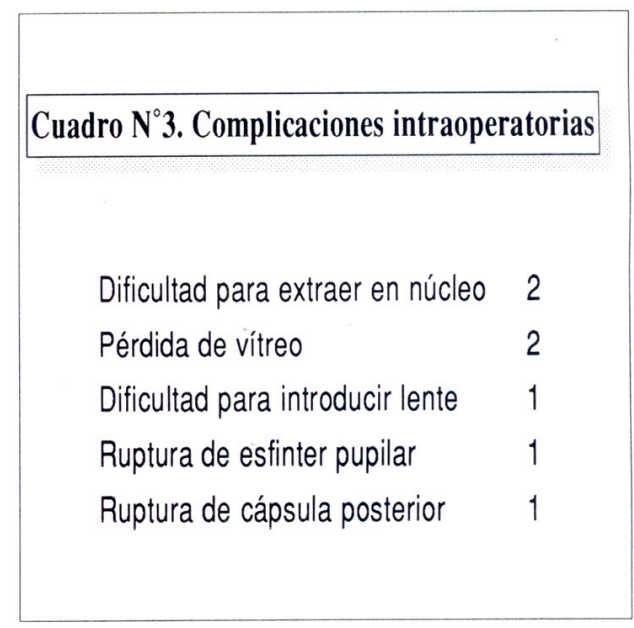

Las complicaciones post-operatorias (Cuadro $\mathrm{N}^{0} 4$ ), también pudieron ser controladas adecuadamente consiguiéndose un buen resultado visual final. La complicación más frecuente fue la queratopatía estriada.

\begin{tabular}{|lrc|}
\hline Cuadro N ${ }^{\circ}$ 4. Complicaciones post-0peratorias \\
\hline Queratopatía estriada & 11 & $(38 \%)$ \\
Edema corneal & 3 & $(10 \%)$ \\
Hipertensión ocular & 3 & $(10 \%)$ \\
Uveitis & 3 & $(10 \%)$ \\
Sinequias posteriores & 3 & $(10 \%)$ \\
Pupila ovalada & 2 & $(7 \%)$ \\
Restos corticales & 2 & $(7 \%)$ \\
Dellen & 1 & $(3 \%)$ \\
Opacificación cápsula posterior & 1 & $(3 \%)$ \\
& & \\
\hline
\end{tabular}

\section{DISCUSIÓN}

De los 29 casos con implante de lente intraocular primario, 6 recibieron in implante de cámara anterior. Este hecho se explica porque los lentes intraoculares fueron donados y ocasionalmente no teníamos el lente de cámara posterior con el poder dióptrico necesario, utilizándose un lente de cámara anterior. Sin embargo, el resultado visual no varió y fueron escogidos midiendo la distancia blanco-blanco lo más exacto posible para evitar astigmatismo por un lente muy grande (3).

Es más importante mencionar que a los pacientes con glaucoma siempre se le implantó un lente de cámara posterior, pués un lente de cámara anterior puede causar alteraciones en el trabéculo, que de por si, puede ya estar alterado, causando mayor elevación de la presión intraocular $(5,10)$. 
La agudeza visual post-operatoria fue buena, consiguiéndose una agudeza visual mayor o igual a $20 / 40$ en el $62 \%$ de los casos y de $20 / 70$ o mejor en el $86 \%$ de ellos. Todos los pacientes que no presentaron patología retinal obtuvieron una mejoría de 3 o más líneas en la cartilla de Snellen. Estos resultados no los podemos comparar con otros similares, publicados por residentes en nuestro medio. Sin embargo, trabajos publicados por residentes norteamericanos obtienen resultados parecidos $(6,13,14)$ y trabajos similares publicados por oftalmólogos de nuestro medio, también muestran coincidencia en sus resultados $(7,8,9)$. Creemos importante recalcar que la mitad de los pacientes $(55 \%)$ con agudeza visual menor de 20/40, tenían algún tipo de patología retinal. Si esto no hubiese ocurrido, el porcentaje de pacientes con agudeza visual mayor o igual a 20/40 podría haberse elevado a $83 \%$.

Una causa de agudeza visual menor de $20 / 40$ en pacientes operados de catarata es el edema macular cistoide subclínico $(4,11)$, cuyo diagnóstico se basa en la angiofluoresceinografía retinal, examen que no se pudo realizar por los bajos recursos económicos de los pacientes de nuestro hospital.

El astigmatismo final post-operatorio se registró sólo en 19 pacientes pués antes no hacíamos corrección astigmática y usábamos Seda Virgen 8/0 para suturar la herida corneal. El astigmatismo post-operatorio se debe a la tracción de las suturas al momento de cerrar la herida esclero-corneal y depende de la tracción que se apliquen en ellas. A mayor tensión, mayor astigmatismo.

El objetivo de la corrección astigmática es disminuir la magnitud del astigmatismo a 2 dioptrías o menos, a los 2 meses post-operatorios (1), mediante el corte selectivo de suturas tal como se mencionó anteriormente. Este astigmatismo deberá localizarse en la zona de la incisión. El objetivo se consiguió en el 79\% de los pacientes. Se busca el valor de 2 dioptrías o menos debido a que con el tiempo, el astigmatismo residual va a disminuir más (aproximadamente hasta los 6 meses post-operatorio), terminando con un astigmatismo cercano a cero o ligeramente contra la regla. De los casos con astigmatismo de 2 dioptrías o menos, el $26 \%$ quedó con un astigmatismo entre 0y 1 dioptría, mientras que el 53\% restante quedó con un astigmatismo entre 1 y 2 dioptrías. Tres pacientes tuvieron un astigmatismo entre 2 y 4 dioptrías y 1 paciente quedó con un astigmatismo mayor de 4 dioptrías (5.75 dioptrías).

En conclusión:

1. La extracción extracapsular con implante de lente intraocular de cámara posterior o anterior, le proporcionan al paciente una recuperación visual satisfactoria.

2. El corte selectivo de suturas reduce considerablemente el astigmatismo postoperatorio, cuando se realiza en forma adecuada y en el tiempo indicado.

3. La evaluación del estado macular y retinal deben ser más exhaustiva en casos de catarata densa, para evitar adelantar pronósticos visuales optimistas y así evitar sorpresas desagradables, tanto para el paciente como para el médico.

4. La cirugía de la catarata con implantes de lente intraocular puede ser realizada por un médico Residente de Tercer Año bajo la supervisión y asesoría de los médicos asistentes.

\section{REFERENCIAS BIBLIOGRÁFICAS}


1.Atkins AD, Roper-Hall MJ. Control of Post-operative astigmatism. Br J Opthalmol 1985; 69: 348.-51.

2.Bradford JD, Wilkinson CP, Bradford RH. Cystoid Macular Edema Following Extracapsular Cataract Extracti and Posterior Chamber Intraocular Lens Implantation. Retina 1988; 8: 161-64.

3.Brown NAP, Sparrow JM. Control of Astigmatism in Cataract Surgery. Br J Ophthalmol 1988; 72: 487-93.

4.Kanski JJ, Packard RBS. Cataract and Lens Implant Surgery. Churchill Livingstone, New York, 1985.

5.Katsumi O, Miyanaga Y, Hirose T, Okumo H, Asaoka I.Binocular Function in Unilateral Aphkia. Ophthalmology 1988; 95: 1088-93.

6.Konner KS, Dulaney Dd, Zimmerman T. Intraocular Preassure Following ECCE and IOL Implantation in Patients with Glaucoma. Ophthalmic Surg 1988; 19: 570-75.

7.Kooner KS, Dulaney DD, Zimmerman TJ. Intraocular Pressure Following Extracapsular Cataract Extraction and Posterior Chamber Intraocular Lens Implantation. Ophthalmic Surg 1988; 19: 471-73.

8.Krupin T, Feitl ME, Bishop KI. Postoperative Intraocular Pressyre Rise in Open-Agle Glaucoma Patients after combined Cataract-filtration Surgery. Ophthalmology 1989; 96: 579-84.

9.Mltzman BA, Haupt EJ, Capiello L, Cinotti DJ. Anterior Chamber Implants and Postoperative Astigmatism. CLAO j 1986; 12: 32-35.

10.Roper-Hall MJ. Atkins AD. Control Astigmatism after Surgery and Trauma: A new Technique . Br J Ophtlalmol 1985; 69: 352-59.

11. Severin TD, Severin SL. Psudophakic Cystoid Macular Edema: A Revised Comprison of the Incidence with Intracapsular and Extracapsular Cataract Extraction. Ophthalmic Surg 1988; 19: 116-18.

12. Tennant JL. A primer of Cataract and IOL. Surgery. Dallas Eye Institute, 1981; Dallas.

13.Kersten RC, Kolder HE, Intraocular Lens Implantation: Resident vs. Staff. Ophthalmic Surg 1982; 13: 470-74.

14.Wong MY, Kline OR. Wills Eye Hospital Residents Intraocular Lens Experience Ophthalmic Surg 1982; 13: 934-35.

15.Engelstein JM. Cirugía de las Cataratas. Editorial Médica Panamericana, Buenos Aires, 1984. 